\title{
Some Statistical Inferences on the Parameters of Records Weibull Distribution Using Entropy
}

\author{
Gholamhossein Yari* and Rezvan Rezaei \\ Iran University of Science and Technology
}

\begin{abstract}
In this paper, we discuss different estimators of the records Weibull distribution parameters and also we apply the Kullback-Leibler divergence of survival function method to estimate record Weibull parameters. Finally, these estimators have been compared using Monte Carlo simulation and suggested good estimators.
\end{abstract}

Keywords. Weibull distribution; upper record; Entropy; parameter estimation, simulation.

MSC 2010: 62N02; 62F10; 62F15; 54C70; 37M05.

\section{Introduction}

In the common practice, the communication theory is necessary in order to quantify and fully study the information mathematically. Information theory is a branch of applied mathematics, electrical engineering and computer science involving the quantification of information. Limited informationtheoretic ideas had been developed by Nyquist (1924), Hartley (1928) and etc. What is known today as the information theory has been founded by Shannon in 1948 through introducing $H(X)$ as the entropy of random variable $X$. He for the first time introduced the qualitative and quantitative model of communication as a statistical process underlying information theory. Later on, other measures were introduced such as Renyi entropy, relative

\footnotetext{
* Corresponding author
} 
entropy and Mutual information. Relative entropy (also Kullback-Leibler divergence) was first defined by Kullback and Leibler in 1951 as the directed divergence between two distributions.

Also, record values and associated statistics have widely been used in many real life applications, for example in sports, weather, business, etc. Record values are very important in case when observations are difficult to obtain or when they are being destroyed when observations are subjected to an experimental test. Observations are obtained by observing successive maximum (minimum) values. The term record value was first introduced by Chandler (1952). Some inferential statistics based on record value have been discussed by Balakrishnan and Chan (1993), Ahmadi and Balakrishnan (2004), Ahmadi and Doostparast (2008), Ahsanullah (1995, 2004) and etc.

In statistics, the Weibull distribution is one of the most important continuous probability distributions. It was, first introduced by Weibull in 1939 when he was studying the issue of structural strength. Teymori and Gupta (2012) introduce a point estimator for shape parameter of upper record Weibull distribution and use maximum-likelihood estimation (MLE) of scale parameter in their method. Also some inferential statistics based on Weibull distribution have been discussed by Yari and Saghafi (2012) and Yari et al. (2013).

In this paper we use Kullbak-Leibler method for estimation parameters of upper record Weibull and we discuss on point estimator method. For estimation $\alpha$ the results show moment method estimation (MME) or KullbackLeibler divergence of survival function estimation (DLS) of $\beta$ are better than maximum-likelihood estimation (MLE) of $\beta$.

Now we introduce some basic definitions that play a central role in the present paper, using the notation of Wuu (2006), Cover and Thomass (2006) and Yari et al. (2013).

Definition 1. Let $Y_{n}=\max \left\{X_{1}, X_{2}, \ldots, X_{n}\right\}, n \geqslant 1$. $X_{i}$ is an upper record of $X_{1}, X_{2}, \ldots, X_{n}$ if $Y_{i}>Y_{i-1}, i \geqslant 1$. By this definition $X_{1}$ is an upper record values.

Since we can replace $X_{1}, X_{2}, \ldots, X_{n}$ by $-X_{1},-X_{2}, \ldots,-X_{n}$ or $X_{1}^{-1}, X_{2}^{-1}, \ldots$, $X_{n}^{-1}$ (if $P\left(X_{i}>0\right)=1$, for all $i$ ), the upper record values of these new sequence will correspond to lower record values of the original sequence, so in this paper we shall study only upper record values and shall use the notation 
$X_{U(i)}$ for the $i$ th upper record statistic. The probability density function (pdf) of the $X_{U(i)}$ is obtained by

$$
f_{X_{U(i)}}(x)=\frac{\left[-\log \left\{1-F_{X}(x)\right\}\right]^{i-1} f_{X}(x)}{\Gamma(i)}, \quad x \in R, \quad i=1,2, \ldots, n,
$$

where $\Gamma$ is the gamma function.

Definition 2. Suppose that $X$ is a random variable with a pdf $f(x)$ and support $S_{x}$, then the entropy of $X$ is defined by

$$
h(X)=-\int_{S} f(x) \log f(x) d x .
$$

Definition 3. Let $X_{1}, X_{2}, \ldots$ be a sequence of positive, independent and identically distributed (iid) random variable with a non-increasing survival function $\bar{F}(x, \Theta)=P_{\Theta}(X>x)$ with support $S_{x}$ and vector of parameters $\Theta$. Then the empirical survival function of a random sample of size $n$ is defined by

$$
G_{n}(x)=\sum_{j=0}^{n-1}\left(1-\frac{j}{n}\right) I_{\left[X_{(j)}, X_{(j+1)}\right)},
$$

where $I$ is the indicator function and $\left(0=X_{(0)} \leqslant\right) X_{(1)} \leqslant X_{(2)} \leqslant \cdots \leqslant X_{(n)}$ is the ordered sample.

Definition 4. Let $\bar{F}(x, \Theta)$ be the true survival function with unknown parameters $\Theta$ and $G_{n}(x)$ be the empirical survival function of a random sample of size $n$ from $F(x, \Theta)$. Then the Kullback-Leibler divergence of Survival function $G_{n}$ and $\bar{F}$ is given by (Ahmadi, 2009)

$$
D L S\left\{G_{n}(x) \| \bar{F}(x)\right\}=\int_{0}^{\infty} G_{n}(x) \ln \frac{G_{n}(x)}{\bar{F}(x)}-\left\{G_{n}(x)-\bar{F}(x)\right\} d x .
$$

The rest of this paper is organized as follows. In the next section we state some properties of record statistics. Section 3 present the upper record value of Weibull distribution and its entropy. Our approch is illustrated in Section 4. Finally, we compare the estimators of parameters of the upper records Weibull distribution by Monte Carlo simulation in Section 5 . 


\section{Some Properties of Record Statistics}

In current section we examine some properties of upper record statistics. See more details in Teimouri and Gupta (2012).

a) Using (1), the chi-square distribution with $2 \mathrm{i}$ degrees of freedom and conversion of $U=-2 \log \{1-F(x)\}$, we have

$$
F_{X}^{-1}\left(1-\exp \left\{-\frac{\chi_{(2 i)}^{2}}{2}\right\}\right) \stackrel{d}{=} X_{U(i)} .
$$

b) The $100(1-\varepsilon) \%$ confidence interval for $X_{U(i)}$ is

$$
\left(F_{X}^{-1}\left(1-\exp \left\{-\frac{\chi_{\left(2 i, \frac{\alpha}{2}\right)}^{2}}{2}\right\}\right), F_{X}^{-1}\left(1-\exp \left\{-\frac{\chi_{\left(2 i, 1-\frac{\alpha}{2}\right)}^{2}}{2}\right\}\right)\right) .
$$

c) The $p$ th quintile for $0<p<1$ of $X_{U(i)}$ is

$$
F_{X_{U(i)}}^{-1}(p)=F_{X}^{-1}\left(1-\exp \left\{-\frac{F_{\chi_{(2 i)}^{2}}^{-1}(p)}{2}\right)\right] .
$$

In the remainder of this specification, we will state a lemma and two theorems which has been presented in the Baratpour et al. (2007).

Lemma 1. (Ahmadi, 2000) For $n \geqslant 1$, we have

$$
\psi(n)=\int x^{n} e^{-x} \log x d x=n !\left(\sum_{i=1}^{n} \frac{1}{i}-\gamma\right),
$$

where $\gamma$ is the Euler's constant.

Theorem 1. Let $X_{1}, X_{2}, \ldots$ be a sequence of iid random variables from cumulative distribution function (cdf) $F(x)$ with pdf $f(x)$ and entropy $H(X)<$ $\infty$. We have

$$
H\left(X_{U(i)}\right)=\sum_{j=1}^{i-1}\left(\log j-\frac{i-1}{j}\right)+(i-1) \gamma-\phi_{f}(i-1), \quad i \geqslant 1
$$

where $\phi_{f}(i)=\int_{0}^{\infty} \frac{z^{i}}{i !} e^{-z} \log f\left(F^{-1}\left(1-e^{-z}\right)\right) d z$. 
Theorem 2. Let $Y$ is a random variable with $c d f F(x)$ and $p d f f(x)$. Under the assumption of Theorem 1, we have:

$$
\begin{aligned}
& \text { i) } H\left(X_{U(i)}\right) \leqslant H\left(X_{U(i)}^{*}\right)-i-B_{i} E\left\{\frac{\log f_{Y}(y)}{1-F_{Y}(y)}\right\}-\log M \text { and } \\
& \text { ii) } H\left(X_{U(i)}\right) \geqslant H\left(X_{U(i)}^{*}\right)-i-\log M,
\end{aligned}
$$

where $M=f(m)<\infty, m$ is the mode of the distribution, $Y=M X$, $X_{U(i)}^{*} \sim \Gamma(i, 1)$ and $B_{i}=\frac{(i-1)^{(i-1)}}{\Gamma(i)} e^{-(i-1)}$.

\section{The Upper Record Value of Weibull Distribu- tion and Its Entropy}

Now we examine the Shannon's Entropy on the upper record of the Weibull distribution. A continuous random variable $X$ is said to be a two-parameter Weibull distribution with shape parameter $\alpha$ and scale parameter $\beta$, denoted by $X \sim W(\alpha, \beta)$, if its $\mathrm{cdf}$ is

$$
F(x)=1-\exp \left\{-\left(\frac{x}{\beta}\right)^{\alpha}\right\}, \quad x>0 .
$$

Now according to Section 2 and $F^{-1}(y)=\beta\left[-\log \left\{1-F_{X}(x)\right\}\right]^{\frac{1}{\alpha}}$ we have:

a)

$$
\begin{gathered}
\beta\left(\frac{\chi_{2 i}^{2}}{2}\right)^{\frac{1}{\alpha}} \stackrel{d}{=} X_{U(i)}, \\
f_{X_{U(i)}}(x)=\frac{1}{\Gamma(i)}\left(\frac{\alpha}{\beta}\right)\left(\frac{x}{\beta}\right)^{\alpha i-1} e^{-\left(\frac{x}{\beta}\right)^{\alpha}}
\end{gathered}
$$

and

$$
\bar{F}_{X_{U(i)}}(x)=\sum_{j=0}^{i-1} \frac{e^{-\left(\frac{x}{\beta}\right)}\left(\frac{x}{\beta}\right)^{j}}{j !} .
$$

b) The $100(1-\varepsilon) \%$ confidence interval for $X_{U(i)}$ is

$$
\left(\beta\left\{\frac{\chi_{\left(2 i, \frac{\varepsilon}{2}\right)}^{2}}{2}\right\}^{\frac{1}{\alpha}}, \beta\left\{\frac{\chi_{\left(2 i, 1-\frac{\varepsilon}{2}\right)}^{2}}{2}\right\}^{\frac{1}{\alpha}}\right) \text {. }
$$


c) The $p$ th quintile for $0<p<1$ of $X_{U(i)}$ is $q_{U(i)}(i)=\beta$.

d) $H\left(X_{U(i)}\right)=\ln \left(\frac{\alpha}{\beta}\right)-i+\sum_{j=1}^{i-1}\left(\log j-\frac{i-1}{j}\right)+(i-1) \gamma+\left(1-\frac{1}{\alpha}\right)$

and

$$
\times\left(\sum_{j=1}^{i-1} \frac{1}{j}-\gamma\right)
$$

$$
\begin{aligned}
D L S\left\{\bar{G}_{n}(x) \| \bar{F}_{n}(x)\right\}= & \sum_{j=1}^{n-1}\left(1-\frac{i}{n}\right) \ln \left(1-\frac{i}{n}\right) \Delta x_{i+1}+\frac{1}{2 n \beta} \sum_{i=1}^{n} x_{(i)}^{2} \\
& -\sum_{i=1}^{n-1}\left(1-\frac{i}{n}\right) \int_{x_{(i)}}^{x_{(i+1)}} \ln \left(\sum_{i=1}^{n-1} \frac{x^{i}}{\beta^{i} i !}\right) d x \\
& -\left\{\bar{x}-\frac{\beta \Gamma\left(n+\frac{1}{\alpha}\right)}{\Gamma(n)}\right\}
\end{aligned}
$$

where $\Delta x_{i}=x_{i+1}-x_{i}, x_{0}=0$ (Lio, 2001).

\section{Estimation of Parameters of the Upper Records Weibull Distribution}

In this paper, we use the notation $i$ th-UW for the $i$ th upper record Weibull distribution and in current section we estimate shape parameter $\alpha$ and scale parameter $\beta$ by five methods. Suppose that $X_{1}, X_{2}, \ldots, X_{n}$ is a random sample from (7) with sample size $n$.

a) Moment method (MME):

Here we provide the MME method of the parameters of a $i$ th-UW distribution. To this purpose, one can show that its mean and variance of the sample are respectively:
(a) $\bar{x}=\frac{\beta \Gamma\left(i+\frac{1}{\alpha}\right)}{\Gamma(i)}$,
(b) $s^{2}=\frac{\beta^{2} \Gamma\left(i+\frac{2}{\alpha}\right)}{\Gamma(i)}$
$\frac{\beta^{2} \Gamma^{2}\left(i+\frac{1}{\alpha}\right)}{\Gamma^{2}(i)}$.

We consider three cases:

- When $\alpha$ is known, then from $(a)$ we have an estimator for $\beta$, denoted 
by $\tilde{\beta}_{\alpha k n o w n}$ as follow

$$
\tilde{\beta}_{\alpha k n o w n}=\frac{\bar{x} \Gamma(i)}{\Gamma\left(i+\frac{1}{\alpha}\right)} .
$$

- When $\beta$ is known, in this case we need to solve $(a)$ with respect to $\alpha$, to yield $\tilde{\alpha}_{\beta k n o w n}$ the following equation

$$
\Gamma\left(i+\frac{1}{\tilde{\alpha}_{\beta \text { known }}}\right)=\frac{\bar{x} \Gamma(i)}{\beta} .
$$

- When both $\alpha$ and $\beta$ are unknown, first we obtain the population coefficient of variation $(\mathrm{CV})$ from $(a)$ and $(b)$ as follow

$$
\frac{\sqrt{\operatorname{var}(X)}}{E(X)}=\frac{\left\{\Gamma(i) \Gamma\left(i+\frac{2}{\alpha}\right)-\Gamma^{2}\left(i+\frac{1}{\alpha}\right)\right\}^{\frac{1}{2}}}{\Gamma\left(i+\frac{1}{\alpha}\right)} .
$$

Then, equating the sample CV with the population $\mathrm{CV}$ (we observe that the population $\mathrm{CV}$ is independent of $\beta$ ) we have

$$
\frac{s}{\bar{x}}=\frac{\left\{\Gamma(i) \Gamma\left(i+\frac{2}{\alpha}\right)-\Gamma^{2}\left(i+\frac{1}{\alpha}\right)\right\}^{\frac{1}{2}}}{\Gamma\left(i+\frac{1}{\alpha}\right)}
$$

where $s^{2}=\frac{1}{n-1} \sum_{i=1}^{n}\left(x_{i}-\bar{x}\right)^{2}$ and $\bar{x}=\frac{1}{n} \sum_{i=1}^{n} x_{i}$. We need to solve (12) to obtain MME of $\alpha$, denoted by $\tilde{\alpha}$. Then substituting $\alpha$ in (10) we have an estimator for $\beta$, denoted by $\tilde{\beta}$.

\section{b) Maximum-likelihood method (MLE):}

Here the maximum likelihood estimators of $i$ th-UW are considered. The log-likelihood function is given by

$$
\ln L(\alpha, \beta) \propto n \ln \left(\frac{\alpha}{\beta}\right)+(\alpha i-1)\left\{\ln \left(\prod_{i=1}^{n} x_{i}\right)-\ln \beta\right\}-\sum_{i=1}^{n}\left(\frac{x_{i}}{\beta}\right)^{\alpha} .
$$

We consider three different cases: 
- $\alpha$ is known: differentiate (13) with respect to $\beta$ then equating to zero and solving with respect to $\beta$. Then the MLE of $\beta$, denoted by $\widehat{\beta}_{M L E}$, will be

$$
\widehat{\beta}_{\mathrm{MLE}}=\left(\frac{\sum_{j=1}^{n} \alpha x_{j}^{\alpha}}{1+n-\alpha i}\right)^{\frac{1}{\alpha}}
$$

- $\beta$ is known: again differentiate (13) with respect to $\alpha$ and then equating by zero and solving with respect to $\alpha$. Then the MLE of $\alpha$, denoted by $\widehat{\alpha}_{M L E}$, will be

$\frac{\partial \ln L}{\partial \alpha}=\frac{n}{\widehat{\alpha}_{M L E}}+i \ln \left(\prod_{i=1}^{n} x_{j}\right)-i \ln \beta-\sum_{i=1}^{n}\left(\frac{x_{j}}{\beta}\right)^{\widehat{\alpha}_{M L E}} \ln \left(\frac{x_{j}}{\beta}\right)=0$.

- Both $\alpha$ and $\beta$ are unknown: in this case, replacing (14) in (13) and then differentiate on it with respect to $\alpha$ then equating to zero and solving with respect to $\alpha$. Here the MLE of $\alpha$, denoted by $\widehat{\alpha}$, will be

$$
\begin{aligned}
& \frac{n}{\alpha}-n \frac{\sum_{j=1}^{n} x_{j}^{\alpha} \ln x_{j}}{\sum_{j=1}^{n} x_{j}^{\alpha}}+\left\{\frac{n i}{\alpha(\alpha i-1+n)}-\frac{n}{\alpha^{2}} \ln (\alpha i-1+n)\right\} \\
& +\sum_{j=1}^{n} i \ln x_{j}-\frac{1}{\alpha^{2}} \ln \left(\alpha \sum_{j=1}^{n} x_{j}^{\alpha}\right)-\left(i-\frac{1}{\alpha}\right)\left(\frac{1}{\alpha}+\frac{\sum_{j=1}^{n} x_{j}^{\alpha} \ln x_{j}}{\sum_{j=1}^{n} x_{j}^{\alpha}}\right) \\
& +\frac{1}{\alpha^{2}} \ln (\alpha i-1+n)+\left(i-\frac{1}{\alpha}\right) \frac{i}{\alpha i-1+n}-\frac{n-1}{\alpha^{2}}=0 .
\end{aligned}
$$

\section{c) Bayesian method:}

Here, we assume the previous information of $\alpha$ and $\beta$ are independent of each other, so $\pi(\alpha, \beta)=\pi(\alpha) \pi(\beta)$. We obtain the Bayes estimator under normal, uniform and triangular prior distributions by Monte Carlo simulation.

\section{d) Point estimator for the shape parameter (PE):}

Theorem 3. (Teimouri and Gupta, 2012) Suppose that a sequence of ith upper record from Weibull family are observed. A simple estimator of shape parameter is given by

$$
\widehat{\alpha}_{P E}=\frac{\log \left(0.000002 i^{2}+0.9998 i-0.3271\right)}{\log \left\{\widehat{m}_{U(i)}\right\}-\log (\beta)},
$$


where $\widehat{m}_{U(i)}$ is the sample median of ith upper record values (Ahsanuullah, 2004).

e) Kullback-Leibler divergence of survival function method (DLS): To estimate the parameters by this method, we set (8) in (4). Then we have

$$
\begin{aligned}
\operatorname{DLS}\left\{G_{n}(x) \| \bar{F}_{n}(x)\right\}= & \sum_{i=1}^{n-1}\left(1-\frac{i}{n}\right) \ln \left(1-\frac{i}{n}\right) \Delta x_{i+1}+\frac{1}{2 n \beta} \sum_{i=1}^{n} x_{(i)}^{2} \\
& -\sum_{i=1}^{n-1}\left(1-\frac{i}{n}\right) \int_{x_{(i)}}^{x_{(i+1)}} \ln \left(\sum_{i=1}^{n} \frac{x^{i}}{\beta^{i} i !}\right) d x \\
& -\left\{\bar{x}_{n}-\frac{\beta \Gamma\left(n+\frac{1}{\alpha}\right)}{\Gamma(n)}\right\}
\end{aligned}
$$

where $\Delta x_{i}=x_{i+1}-x_{i}, x_{0}=0$. Now differentiate (18) with respect to $\alpha$ then equating to zero and solving with respect to $\alpha$. Then, $\frac{\partial}{\partial \alpha} \Gamma\left(i+\frac{1}{\alpha}\right)=0$. But we don't have a good estimator for $\alpha$ by this equation since it does not depend on the sample values. Now we suppose that $\alpha$ is known. The equation (18) should be minimized respect to $\beta$. For this propose, we have

$$
\frac{\Gamma\left(i+\frac{1}{\alpha}\right)}{\Gamma(i)}-\frac{\sum_{i=1}^{n} x_{(j)}^{2}}{2 n \beta^{2}}-\sum_{l=1}^{n}\left(1-\frac{l-1}{n}\right) \int_{x_{(l-1)}}^{x_{(l)}} \frac{\sum_{k=0}^{n-1} \frac{\left(\frac{x}{\beta}\right)^{k}}{(k-1) !}}{\sum_{k=0}^{n-1} \frac{\left(\frac{x}{\beta}\right)^{k}}{k !}} d x=0 .
$$

We observe that a close form solution of (19) for $\beta$ is not possible.

\section{Simulation Study}

Since the MME, MLE, DLS and Bayesian estimate of the parameters have not closed form so checking the performance of them, theoretically is a difficult task. Therefore, they must be solved numerically. We have done this work by Matlab software. First, we have generated $x_{1}, x_{2}, \ldots, x_{n}$ from a $i$ th $=4$ upper record Weibull distribution (for more details, see Teymori and Gupta, 2012). In this study we assumed $\alpha_{\text {true }}=2, \beta_{\text {true }}=3$ and both $\alpha$ and $\beta$ are unknown. This sample simulated was used to estimate the 4 th-UW using the MME, MLE, DLS, Baysian and point methods. The above process was repeated 10,000 times. Consequently, we have a set of $M=100004$ thUW parameter estimates using each method. Then the mean values $\bar{\alpha}$ and 
$\bar{\beta}$, and sample variances $S_{\alpha}^{2}$ and $S_{\beta}^{2}$ and mean squered errors values $\mathrm{MSE}_{\alpha}$ and $\operatorname{MSE}_{\beta}$ were computed using:

$$
\begin{gathered}
\bar{\alpha}=\frac{1}{M} \sum_{k=1}^{M} \alpha_{k}, \quad \bar{\beta}=\frac{1}{M} \sum_{k=1}^{M} \beta_{k}, \\
S_{\alpha}^{2}=\frac{1}{M-1} \sum_{k=1}^{M}\left(\alpha_{k}-\bar{\alpha}\right)^{2}, \quad S_{\beta}^{2}=\frac{1}{M-1} \sum_{k=1}^{M}\left(\beta_{k}-\bar{\beta}\right)^{2}, \\
\operatorname{MSE}_{\alpha}=S_{\alpha}^{2}+\left(\bar{\alpha}-\alpha_{\text {true }}\right)^{2}, \\
\operatorname{MSE}_{\beta}=S_{\beta}^{2}+\left(\bar{\beta}-\beta_{\text {true }}\right)^{2},
\end{gathered}
$$

where $\alpha_{k}, \beta_{k}$ are the estimated 4th-UW shape and scale parameters from $k$ th sample. To illustrate the effect of sample size, we carried out a simulation study for some levels of $n=5,6, \ldots, 30$. First, we study the behavior of $\beta$ given in (10), (14), (19) and Bayesian method with four prior distribution. Figure 1 shows the simulation results of $\beta$. The following results can be concluded from this figure:
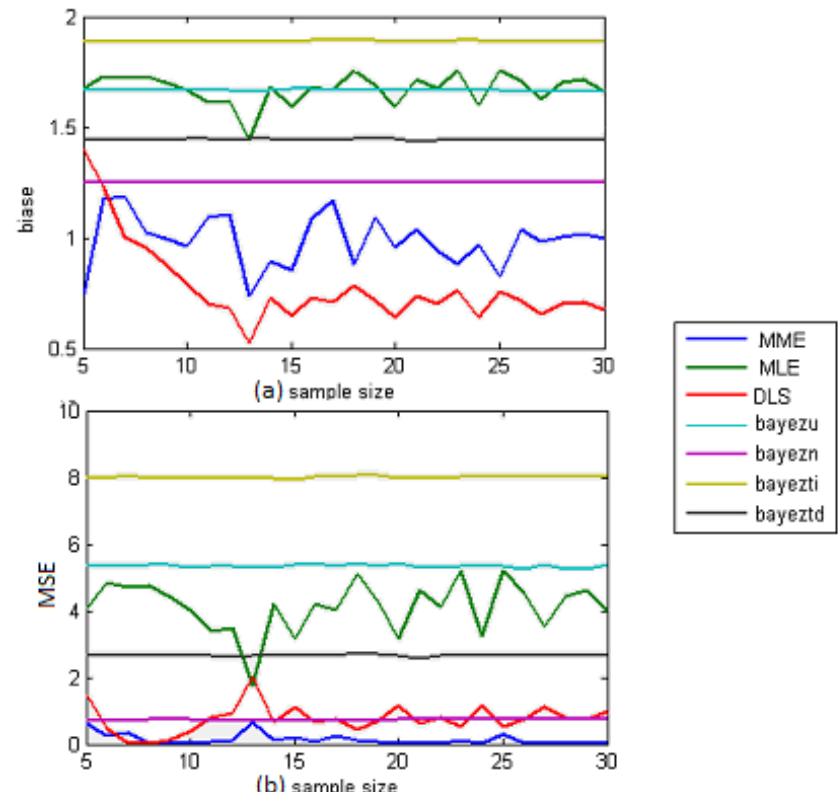

Figure 1. (a) $\frac{\bar{\beta}}{\beta}$. (b) $\widehat{M S E}_{\beta}$ as functions of sample size. 

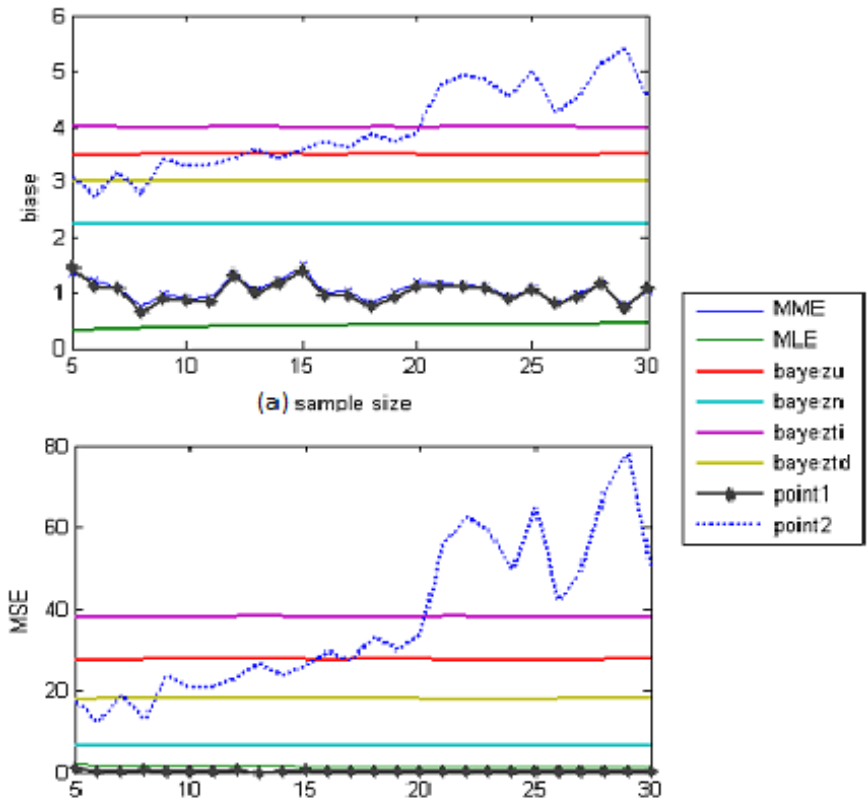

(b) sample size

Figure 2. (a) $\frac{\bar{\alpha}}{\alpha}$. (b) $\widehat{M S E_{\alpha}}$ as functions of sample size.

1) According to Figure 1.a, the MME, DLS and Bayesian with normal prior methods act better than the rest in terms of bias. MME and Bayesian estimate for $\beta$ are overestimated whereas DLS is underestimated.

2) According to Figure 1.b, mean squared error (MSE) of MME method is best for every $n$.

Now we study the behavior of $\alpha$ given in (12), (16), (17) and Bayesian method with four prior distribution. Figure 2 shows the simulation results for $\alpha$. The following results can be concluded from this figure:

1) According to Figure 2.a, the MME and point estimator methods act better than the rest and are so similar in terms of bias. Also MLE values for $\alpha$ are closed to $\alpha_{\text {true }}$, so this method can be a good estimator for $\alpha$. 
2) Figure 2.b shows MSE of MME, MLE and point estimator methods are best for every $n$ and very closed to zero.

\section{Conclusion}

The current paper concerned with different estimators of the records Weibull distribution parameters and discussed how appropriate and inappropriate these estimators are. The simulation process, suggests MME and DLS methods for estimating the parameter $\beta$. MME and proposed point estimators based on estimated $\beta$ by MME indicated to be appropriate estimators for $\alpha$.

\section{References}

Ahmadi, J. and Balakrishnan, N. (2004). Confidence Intervals for Quantiles in Terms of Record Range. Statistics 83 probability Letters, 68, 395-405.

Ahmadi, J. and Doostparast, M. (2008). Satistical Inference Based on K-records, Mashhad R. J. Math. Sci., 1, 67-82.

Ahmadi, J. (2009). Entropy Properties of Certain Record Statistics and Some Characterization Results, JIRSS, 7, Nos. 1-2, 1-13.

Ahsanullah, M. (1995). Record Statistics, Nova Science Publishers, Inc., New York.

Ahsanullah, M. (2004). Record Values-Theory and Applications, University Press of America, Inc., New York.

Balakrishnan, N. and Chan, P.S. (1993). Record Values from Rayleigh and Weibull Distribution and Associated Inference. proceedings of the confidence on Extreme Value Theory and applications, volume 3 Gaithersbarg, Maryland.

Baratpour, J., Ahmaidi, J. and Arghami, R. (2007). Entropy Properties of Record Statistics, Statistical Papers, 48, 197-213.

Chandler, K.N. (1952). The Distribution and Frequency of Record Values, Journal of the Royal Statistical Society, Series B, 14, 220-228.

Cover, T.M. and Thomas, J.A. (2006). Elements of Information Theory, 2nd ed., Wiley, New york.

Hartley, R.V. (1928). Transmission of Information. Bell Syst. Tech. J., 7, 535.

Kullback, S. and Leibler, R.A. (1951). On Information and Sufficiency. Ann. Math. Stat., 22, 79-86. 
Nyquist, H. (1924). Certain Factors Affecting Telegraph Speed. Bell Syst. Tech. J., 3, 324.

Shannon, C.E. (1948). A Mathematical Theory of Communication. Bell Syst. Tech. J., 27, 379-423, 623-656.

Teimouri, M. and Gupta, A.K. (2012). On the Weibull Record Statistics and Associated Inferences, statistica, anno LXXII, n.2.

Wuu Wu, J. and Chiao, T. (2006). Satistical Inference about the Shape Parameter of the Weibull Distribution by Upper Record Values, Statistical Papers, 48, 95-129.

Yari, G.H. and Saghafi, A. (2012). Unbiased Weibull Modulus Estimation Using Differential Cumulative Entropy, Communication in Statistics Simulation and Computation, 41, 1-7.

Yari, G.H., Mirhabibi, A. and Saghafi, A. (2013). Estimation of the Weibull Parameters by Kullback-Leibler Divergence of Survival Functions, Appl. Math. Inf. Sci., 7, 187-192.

\section{Gholamhissein Yari}

Department of Statistics,

School of Mathematics,

Iran University of Science and Technology,

Tehran, Iran.

email: yari@iust.ac.ir

\author{
Rezvan Rezaei \\ Department of Statistics, \\ School of Mathematics, \\ Iran University of Science and Technology, \\ Tehran, Iran. \\ email:rezvan_rezaei@mathdep.iust.ac.ir
}


